\title{
Sentiment-based influence detection on Twitter
}

\author{
Carolina Bigonha $\cdot$ Thiago N. C. Cardoso • \\ Mirella M. Moro • Marcos A. Gonçalves • \\ Virgílio A. F. Almeida
}

Received: 14 June 2011 / Accepted: 25 November 2011 / Published online: 24 December 2011

(C) The Brazilian Computer Society 2011

\begin{abstract}
The user generated content available in online communities is easy to create and consume. Lately, it also became strategically important to companies interested in obtaining population feedback on products, merchandising, etc. One of the most important online communities is Twitter: recent statistics report 65 million new tweets each day. However, processing this amount of data is very costly and a big portion of the content is simply not useful for strategic analysis. Thus, in order to filter the data to be analyzed, we propose a new method for ranking the most influential users in Twitter. Our approach is based on a combination of the user position in networks that emerge from Twitter relations, the polarity of her opinions and the textual quality of her tweets. Our experimental evaluation shows that our approach can successfully identify some of the most influential users and that interactions between users provide the best evidence to determine user influence.
\end{abstract}

Keywords Twitter · User influence

\author{
C. Bigonha $(\bowtie)$ T.N.C. Cardoso • M.M. Moro • \\ M.A. Gonçalves · V.A.F. Almeida \\ Departamento de Ciência da Computação, Universidade Federal \\ de Minas Gerais, Belo Horizonte, MG, Brazil \\ e-mail: carolb@dcc.ufmg.br \\ T.N.C. Cardoso \\ e-mail: thiagon@dcc.ufmg.br \\ M.M. Moro \\ e-mail: mirella@dcc.ufmg.br \\ M.A. Gonçalves \\ e-mail: mgoncalv@dcc.ufmg.br \\ V.A.F. Almeida \\ e-mail: virgilio@dcc.ufmg.br
}

\section{Introduction}

Twitter is a micro-blogging tool that represents a real-time information network. Motivated by the question "What's happening?", users of Twitter post messages of up to 140 characters, called statuses, or more familiarly, tweets. A tweet may contain more than just pure text; it may include links to websites, photos, videos and other media, as well short strings preceded by a hash symbol (\#), called hashtags, usually employed to filter or promote content [17]. Also, tweets may refer to other users by preceding their names with an at mark (@). Each Twitter user has a profile page, which contains personal information about her (name, photo, location, etc.), some quantitative data (her number of followers and following users) and her timeline, i.e. a list of tweets that she has posted (public or private, according to the user's decision). Furthermore, a user may follow another by choosing to receive the tweets she posts.

Among many other Online Social Networks, such as Facebook, Orkut, Flickr and Youtube, ${ }^{1}$ Twitter stands out for its simplicity and diversity. Due to the message short size and the effortless posting/reading from anywhere, it is easy to both produce and consume content. Twitter also plays a major role in electronic word of mouth ${ }^{2}$ [20] due to its immediacy of posting (e.g., one can send a tweet at the moment of a purchase or a problem in the bank) and the simplicity of finding out what people are talking about. In summary, users share opinions, experiences and suggestions in large scale. Considering Twitter users as potential con-

\footnotetext{
${ }^{1}$ http://www.facebook.com, http://www.orkut.com, http://www.flickr. com, http://www.youtube.com.

${ }^{2}$ Word of mouth is the process of transferring information (attitudes, opinions about products) from person to person.
} 
sumers/voters, micro-blogging networks have become a rich source of data in any situation in which feedback is desired.

Previous work [26] has also shown that text streams (such as Twitter) are a potential substitute and supplement for traditional public opinion surveys. Therefore, businesses have recently learned the importance of understanding and properly reacting to the information available in Twitter. By analyzing the data and the users, they aim to gather market intelligence and improve their campaigns, products or services acceptance.

However, a huge amount of content is generated daily: on an average day, Twitter publishes about 750 tweets-persecond (tps) whereas on a deciding game of a championship (such as NBA), about 3,000 tps are registered. ${ }^{3}$ Besides being impractical to inspect all the data generated daily (even for a specific topic), not all tweets and users are worth such an evaluation. Under these circumstances, it is crucial to find the key opinion leaders, or influential users, who drive the positive and, specially, the negative conversations on Twitter.

Katz et al. [21] defined as opinion leaders "the individuals who were likely to influence other persons in their immediate environment". Although some may question the existence of influentials [31], its presence and importance are widely discussed in the marketing environment $[4,5,10,30]$. Thus, assuming the existence of such influential users, we propose an approach for finding them in a topic-based scenario. To focus on topics is a matter of design: people are often interested in monitoring one particular topic or context (a product, a personality, an event) [29]. Moreover, focusing on one subject allows us to use sentiment as a measure of user engagement: another influence indicator [14].

We present a method for identifying influential users based on three perspectives: $(\alpha)$ polarity, $(\beta)$ network and $(\gamma)$ quality. Specifically, the polarity perspective considers the classification of the tweets of each user as positive, neutral or negative in order to find the confident positive and negative users. Such a classification allows us to identify what we call evangelists and detractors - influential users who stand in favor or against the subject. The network perspective measures the relation between the user and her neighbors', including actions (re-tweets, replies, mentions). Finally, the quality perspective is used to rate higher users that have well written tweets.

For testing our techniques, we built two datasets for specific topics (two product brands). Each tweet and user data were manually classified as positive/negative/neutral and evangelist/detractor/irrelevant by marketing professionals.

\footnotetext{
${ }^{3}$ http://blog.twitter.com/2010/06/big-goals-big-game-big-records. html.
}

Our experimental results demonstrate that we can successfully identify some of the most influential users concerning a subject using our techniques and that interactions between users are the best evidence to determine user influence. The experiments were performed in diverse topicspecific scenarios, demonstrating the applicability of the method to any subject. Moreover, we show that the topicspecific datasets employed have similar characteristics when compared to some more general Twitter collections used in previous work, such as [18] and [22], meaning that most of our results are potentially generalizable.

The main contributions of this paper are summarized as follows: (i) a definition for influential users on Twitter, which considers the importance of the user within the interactions concerning a topic, the quality of her tweets and her polarity as new indicators of influence; (ii) a method to find the influentials based on the aforementioned concept; (iii) the construction of two datasets for influence experiments, validated by specialists in marketing; (iv) an experimental validation and evaluation of the proposed technique, including tests on two datasets, two naive baselines, analysis of the impact of each view on the result and comparison of the results using interactions via tweets or the followingfollower connections.

This article is organized as follows: Sect. 2 presents a review of the related work; Sect. 3 describes SaID, our influence detection method, including details for the preprocessing phase Sect. 3.1 and metrics analysis Sect. 3.2; Sect. 4 describes the datasets used for testing the proposed technique Sect. 4.1) and discusses the evaluation and validation of the method Sect. 4.2; and, finally, Sect. 5 reviews our main contributions and results.

\section{Related work}

Finding influential users on Twitter has recently attracted much interest. The report presented in [24] highlights interactions (replies, retweets, mentions and attributions) as markers of influence, rather than solely the number of followers. The authors select a few famous users belonging to the categories "celebrity", "news outlet" and "social media analyst" and compare several influence indicators, e.g., average content spread per tweet, for each user.

A method for topic-sensitive influential users detection is defined in [32]. Considering a Pagerank [8] alike metric, it calculates the user influence based on how many people have received her tweets. In [9], influence is divided in three types: the in-degree influence (the number of followers that a user has), the re-tweet influence (the number of re-tweets containing ones name), and mention influence (the number of times a user is mentioned). The authors study the dynamics of influence across topics and time, analyzing whether 


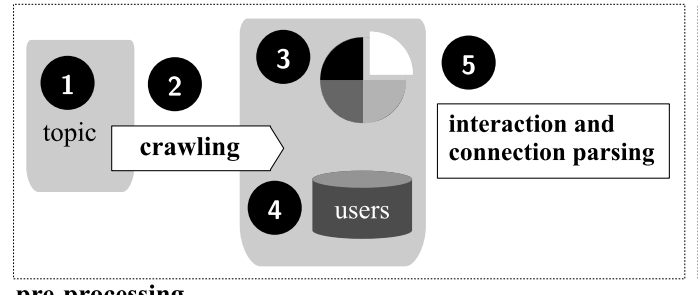

pre-processing

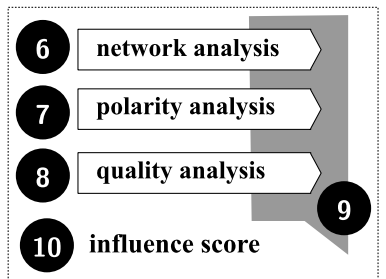

metrics analysis
Fig. 1 SaID workflow: (1) topic definition; (2) crawl of topic-related tweets; (3) sentiment analysis of tweets; (4) authors identification; (5) interaction and connection relations parsing; (6), (7) and (8) net-

users can hold significant influence over a variety of topics, and examining the rise and fall of influentials over time.

Based on the concept that influence is measured by the replication of already performed actions, Goyal et al. [15] propose a technique for constructing influence probability graphs from social networks (friendship graph) and action logs. From these two sources of data, the authors build a propagation graph (where nodes are the users who perform the actions and the edges represent the direction of the propagation), apply models of influence (static, discrete and continuous time) and finally construct the graph of influence probabilities. Both Goyal et al. [15] and Lee et al. [25] emphasize the temporal aspect of influence detection, which is indicated as future work of the presented paper.

In [3], the authors measure influence based on the user's ability to spread brand new content. Given a propagation path traced from the user that created the content (URL) to the last user that received it, they identify the users who are nearer to the origin as the most influent. The attributes considered for the calculation of influence are: the number of followers, number of followings, number of tweets posted and date the user joined Twitter. The authors also analyzed the content of the links posted, observing the average cascade size for different interest ratings, types and categories of posts.

Despite focusing mainly on the topological characteristics of Twitter and its power as an information sharing environment, Kwak et al. [23], compare three methods for ranking users: the first strategy ranks users by the number of followers, the second applies PageRank to a network of followings and followers and the third one ranks users according to the number of her re-tweets. As conclusion, the authors find the same gap between the number of followers and the popularity of one's tweets indicated before.

Our contributions in this article stand out from previous work in key aspects. First, SaID considers more complete metrics for measuring the repercussion of user's actions: we evaluate features of users within an interaction network that captures all the conversations about a topic. Second, we are the first to apply a tweet content quality analysis: our hy- work, polarity and quality analysis, respectively; (9) combination of the metrics into an influence score; (10) rank construction

pothesis is that users who create well written and more understandable tweets are more likely to be influential than others. Also, we evaluate the commitment of the user with the topic, that is, if she is confident positive or negatively and with what frequency. This allows our method to identify the potential evangelists and detractors concerning the topic. Finally, no previous work evaluates its method using a specialists' ground truth. Instead of generating various ranked lists and simply comparing them, we validate our technique based on marketing and communication specialists' point of view.

\section{Influential users identification}

In this article, we present a method, called SaID (Sentimentbased Influence Detection on Twitter) for identifying influential users on Twitter, which relies mainly on their behavior. Figure 1 shows an overview of the proposed method. The two main phases (pre-processing and metrics analysis) are explained in the following sections.

\subsection{Pre-processing}

The pre-processing phase consists of five steps. The first one is determining the topic and time interval; the second is crawling; the third one is the sentiment analysis; the fourth is the extraction of user data; and, at last, the fifth consists on the interaction and connection parsing. This section describes each one of them.

Topic definition In the marketing environment (considering business owners, investors and advertising agencies, for example) the interest is directed to a topic-restricted analysis of influence rather than a global one. An important biologist is possibly not as influent as a politics-engaged user when it comes to discussing this year's election. Under those circumstances, this work evaluates users' influence factors considering topic-related scenarios. Thus, the first step in the pre-processing phase is to determine the topic to 
Table 1 Example of positive, negative and neutral tweets

\begin{tabular}{ll}
\hline positive & "I been using PayPal since 1994. It's the best!" \\
negative & "Got to love paypal. You sell an item, the person gets \\
& it, leaves you positive feedback and then asks paypal \\
& to refund the money and they do." \\
neutral & "Our facebook page is now linked to PayPal so you \\
& can make your tax deductible donation!!"
\end{tabular}

be analyzed. It may be a brand, a product, a personality, an event, and so on. Based on the chosen topic, keyword-based queries are built in this phase.

Crawling There is no established benchmark for evaluating user influence detection on Twitter. So, a major effort of this work is to build the data sets. Although expensive and demanding, this process is essential for the experimental validation presented in Sect. 4. For collecting the data concerning the chosen topic, we use the Twitter API. ${ }^{4}$ Every tweet, publicly available from the user's timeline, which contains the defined keywords, during a certain time interval, is stored. Also, we carefully eliminate retrieved tweets that fit into a different context or have an undesired content (e.g. posts concerning "house", the human habitat, on a search for "House", the TV series).

Sentiment analysis In the third step, every tweet on the dataset is classified either as positive, negative or neutral. Positive ones promote the chosen topic, by expressing user appreciation or satisfaction. Likewise, negative ones express aversion toward the topic and may contain complaints, bad reviews, and so forth. Neutral tweets, on the other hand, are usually the ones that contain unbiased opinions or a purely informative content. Table 1 contains tweets for each sentiment concerning PayPal (an online service for payments and money transfers). This example also emphasizes the complexity of classifying tweets' sentiment. Aside from its short size, its content is often colloquial and filled with irony and sarcasm, both tones that are hard to identify. Note that, in Table 1 , the negative tweet is only negative due to the last three words "and they do".

In this work, the tweets were manually classified by a marketing analysts' team, in a process in which each tweet's sentiment was verified at least by two analysts and a supervisor. In case of disagreement, the supervisor's decision was taken into account. ${ }^{5}$ This sentiment analysis allows the detection of engagement of the users toward the defined topic and, consequently, leads to the identifying users who, besides from being well connected regarding interactions, are

\footnotetext{
${ }^{4}$ http://dev.twitter.com/.

${ }^{5}$ The automatization of this step and the measurement of its impact on the proposed technique is one of the main focuses of our current research.
}

responsible for influencing other's decisions due to the polarity of their tweets. Furthermore, in a "crisis management" point of view, to recognize the users who lead the positive and, mainly, the negative information flow is essential.

User data extraction As already mentioned, our method gathers the content generated on Twitter via tweets that mention a certain keyword set. Since our interest is on user's characterization, we must identify the author of each tweet and collect her information (using the Twitter API). We store author's name and her list of followers and following users.

Interaction and connection parsing Finally, the last step in the pre-processing phase is executed, in order to extract the interactions and connections between users. It is very common for a user to interact with others in a post by using the '@' notation prefacing their username. We acknowledge four types of possible interaction via tweets: replies, retweets, mentions and attribution. A reply corresponds to a situation in which one user wants to answer a post from another user or simply direct the message to someone else. For example, a tweet of user $A$ in reply to user $B$ would be a post like '@B [content of the tweet]'. A retweet is used to propagate a message: $A$ retweets $B$ means that $A$ posted a message that $B$ has already posted. Retweets, particularly, either have a "RT" markup_for example, 'RT @B [content posted by B]'-or have a Twitter official retweet identification. Finally, a mention is a tweet that contains another user in the middle of the text (e.g. '[content] @ A [content]') and an attribution is similar to a retweet, except that it cites the username using the notation '(via @B)' instead of 'RT @B'. We parse each gathered tweet and store all the interactions for further analysis. Finally, we extract all the followerfollowing relations between the users in the dataset, based on each users' friends list gathered in the previous step.

\subsection{Influence metrics analysis}

The second phase is the actual influence analysis, in which network, polarity and quality values are calculated and combined into a single factor, as explained further.

\subsubsection{Network analysis}

In order to characterize the roles of users on Twitter and identify the influential ones, we first adopt a complex network approach. From the several networks that naturally emerge from user relations enabled by Twitter features, we select two of them for an in-depth analysis: the Connection Graph $\left(G_{c}\right)$ and the Interaction Graph $\left(G_{i}\right)$. Intuitively, the first network captures the declared connections between users (following-follower relation) whereas the second one captures the user interactions via tweets. Formally, the networks are defined as follows. 
Definition 1 (Connection Graph) For a given subset of users involved in a specific theme, let $\left(G_{c}, U\right)$ be the user directed unweighted graph, where $\left(u_{1}, u_{2}\right)$ is a directed arc in $U$ if user $u_{1} \in G_{c}$ follows user $u_{2} \in G_{c}$.

Definition 2 (Interaction Graph) For a given subset of users involved in a specific theme, let $\left(G_{i}, U\right)$ be the user directed unweighted graph, where $\left(u_{1}, u_{2}\right)$ is a directed arc in $U$ if user $u_{1} \in G_{i}$ has cited at least once (i.e., mention, reply or re-tweet) user $u_{2} \in G_{i}$.

From the different measures for network analysis that could be exploited, such as shortest paths, distance, component connectivity, clustering, clique, among others [12], the measurements that make more sense for influence estimation are those based on centrality, defined on the vertices of a graph. These metrics are designed to rank the notoriety of users according to their position in the network. Similarly, influential users have to be well connected to other users, and play a central role in the graph in which she is embedded. For that matter, two centrality measures were chosen. Furthermore, we analyse the in-degree of the users ${ }^{6}$, as follows.

- Betweenness centrality $(b c)$ is the first centrality measure, and is defined by the fraction of shortest paths between node pairs that pass through the node of interest [7]. In both graphs $G_{i}$ and $G_{c}$, users with high betweenness have an important role in the information dissemination process, since they act as bridges for the data flow.

- The centrality measure Eigenvector centrality $(e c)[6,28]$ considers that an user is more central if she is related to users that are themselves central. Thus, the centrality of some node does not only depend on the number of its adjacent nodes, but also on their value of centrality. It is important to remark that Eigenvector centrality is an algorithm similar to Pagerank, applied to social networks [11]. We use this metric to rank higher users that are related with many other users or with a few users that are related with lots of other users.

- The In-degree (id) of each user is a key characteristic of the structure of a directed network. In the Interaction Graph, the in-degree measures the number of times a user was cited or had her tweets replied or retweeted, whereas in the Connection Graph, the in-degree stands out for the number of users within the topic that follows the user in focus. $^{7}$

\footnotetext{
${ }^{6}$ All metrics were calculated using Networkx [16].

${ }^{7}$ In the Connection Graph, the in and out-degrees of each user is different from the number of following and follower users that appear on her profile, because they concern the connections between the users within the collected dataset.
}

Besides these network features, we also employ the Twitter Follower-Followee Ratio (TFF). This metric can be useful to characterize the user, as presented in [22, 24], thus, representing a good influence indicator. According to $[22,24]$, if the ratio approaches infinity ( $\uparrow$ followers, $\downarrow$ following), the user is likely to be a "broadcaster", such as news media profiles, celebrities or other popular users. On the other hand, if the ratio approaches 1 (followers $\simeq$ followees), the user has reciprocity on her connections. This describes the most common types of user. Finally, if the ratio approaches zero ( $\downarrow$ followers, $\uparrow$ followees), the user might be categorized as a spammer or a robot, which follows way more users than is followed by (people do not usually follow back spammers/robots). Based on this characteristics, TFF is presented as an additional metric for studying the collected data. We use this metric, combined with others, to identify influential users in our dataset, considering the users with higher TFF as more relevant. This metric helps eliminating potential spammers (that may fit in the second and third groups) and valorize the users that are widely followed, but have some selection for following others.

From an influence detection point of view, the most influential user in a database, would be the one with higher value for each of the four metrics aforementioned $(b c, e c, i d, t f f)$. For this reason, the metrics were combined in an arithmetic mean (as shown in (1)):

$u_{\text {network }}=(b c+e c+i d+t f f) / 4$.

In order to combine them equally, they were normalized ${ }^{8}$ individually to a $[0,1]$ scale [19]. The result $u_{\text {network }}$ is also in this range. Due to the broad distribution of centrality measure values, the normalization of ec and bc was calculated using logarithmic quantities.

\subsubsection{Polarity analysis}

The next perspective of influence analysis corresponds to the author's polarity. This perspective value is calculated based on the classification of tweets, performed in the preprocessing phase. For each user, it considers her overall contribution to the topic discussion: if she posts mostly positivebiased content, she is a potential evangelist. On the other hand, if she posts mostly negative-biased content, she is a potential detractor. Users that stay in the middle are neutral. We consider that positive and negative tweets nullify each other. Thus, for each user, the polarity value is the summa-

\footnotetext{
${ }^{8}$ Specifically, we did a Range Normalization [19], in which the range is changed from $\left[x_{\min }, x_{\max }\right]$ to $[0,1]$. The scaling formula is $x_{i}^{\prime}=$ $\frac{x_{i}-x_{\min }}{x_{\max }-x_{\min }}$, where $\left\{x_{1}, x_{2}, \ldots, x_{m}\right\}$ are the measured values and $x_{i}^{\prime}$ the $x_{\max }-x_{\min }$
scaled value corresponding to $x_{i}$.
} 
tion of the sentiment of all her tweets, as shown in (2):

$u_{\text {polarity }}=\sum_{i=1}^{i \leq n_{u}} t_{i}, \quad$ where $t_{i}= \begin{cases}w_{+} & \text {if } t_{i} \text { is positive } \\ w_{0} & \text { if } t_{i} \text { is neutral } \\ w_{-} & \text {if } t_{i} \text { is negative }\end{cases}$

In the formula, $t_{i}$ is the $i$ th tweet (of $n_{u}$ total tweets) of user $u$ and $w_{+}, w_{0}$ and $w_{-}$are the weights associated with positive, neutral and negative tweets, respectively. The weight is used for balancing the sentiments. For example, one may want to increase the weight of negative tweets to highlight detractors. Also, one may argue that if a user made the effort to write a non-negative tweet on the topic, she is positively contributing to the spread of news about the subject, thus neutral and positive tweets are the same. In this article, following the specialists' instructions, we considered that there are three classes of tweet sentiment and that the neutral ones contribute (with lower intensity) to the user's positive polarity, by using weights $w_{+}=+2, w_{-}=-2$ and $w_{0}=+1$. Similarly to the network perspective, the polarity values were range normalized: positive values to $[0,1]$ and negative values to $[-1,0]$.

\subsubsection{Quality analysis}

At last, we analyze the content of the tweet itself. User generated content is usually very heterogeneous, due to the variety of users' background and their different intentions. Our goal in analyzing the quality of the tweet content is to rank higher posts (and, consequently, their authors) that are well written and understandable. We hypothesize that if a user is to influence other people, her tweets are expected to have a minimum quality. For that matter, each tweet is evaluated using the Flesch-Kincaid Grade Level metric [27] (kincaid $_{i}$ ), which was designed to indicate comprehension difficulty when reading a passage of contemporary academic English. This metric, successfully applied in the identification of high-quality Wikipedia articles [13], increased the accuracy of the influential identification for some cases, as studied in the experiments in Sect. 4.2. For each tweet, it computes the average number of syllables per word and the average sentence length. For example, a tweet like "aaaaaaa haaate justin bieber!" would have a low quality value, while "PayPal is dangerously easy." a high one. The user quality perspective was determined as the average of the Kincaid metric computed for each one of her tweets, as defined in (3), using the package Style and Diction. ${ }^{9}$

$u_{\text {quality }}=\sum_{i=1}^{i \leq n_{u}}$ kincaid $_{i} \times \frac{1}{n_{u}}$.

${ }^{9}$ http://www.gnu.org/software/diction/diction.html.

\subsubsection{Influence score}

So far, we have presented different types of information that can help characterizing Twitter users, divided into three perspectives: polarity, network and quality. By exploiting them together, we can obtain a user ranking and assign a single value (influence score) to each user. The user rank is given by (4) and is one of the main contributions of this work.

$I_{s}=\frac{\alpha \cdot u_{\text {polarity }}+\varphi \cdot\left(\beta \cdot u_{\text {network }}+\gamma \cdot u_{\text {quality }}\right)}{\alpha+\beta+\gamma}$,

where

$u_{\text {polarity }}, u_{\text {network }}, u_{\text {quality }}$ are the normalized polarity, network and quality perspectives;

$\alpha, \beta, \gamma$ are constants, greater or equal to zero, that weight each of the three perspectives; and

$\varphi=\frac{u_{\text {polarity }}}{\left|u_{\text {polarity }}\right|}$.

As aforementioned, both network and quality perspective values were normalized to fit into the range $[0,1]$, whereas the polarity perspective values fit into $[-1,1]$. The auxiliary variable $\varphi$ adjusts both network and quality perspectives according to the polarity result. If a user has a polarity equal to zero, the result of the equation is zero (regardless of the other features). Also, if the polarity is negative, both network and quality have their signal changed. The resulting influence score, for each user, is in the range $[-1,1]$. By sorting the users in descending order, the top ones, with $I_{S}>0$, are evangelists or neutral users and the bottom ones, with $I_{s}<0$, detractors.

The idea behind combining different perspectives into a single influence score is that a feature alone may not be enough to characterize whether a user is influent or not, whereas the combination of the features may be. A user that is well connected in the graph, has a biased opinion, and writes high quality tweets should be ranked higher as an influential user. The formula eliminates types of profile that are erroneously appointed as influent. For example: (i) someone that is well connected, but does not have biased opinion about the subject; (ii) someone that posts daily hundreds of positive/negative tweets about the topic, but, for any reason, no one pays any attention to; (iii) a person whose content is too noisy and does not have a persuasive speech. For the specific cases listed above, the low values of polarity (i), network (ii) and quality (iii), respectively, would keep the users from being considered as influent.

\section{Experiments and discussion}

This section introduces the datasets applied to evaluate our approach (Sect. 4.1) along with the experiments, the results and a discussion (Sect. 4.2). 


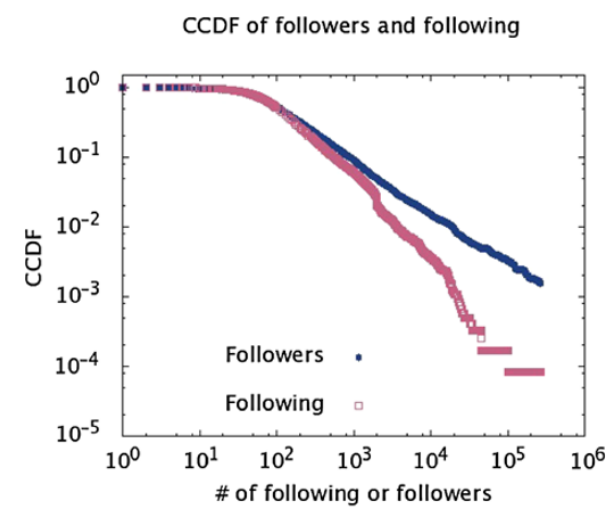

(a)

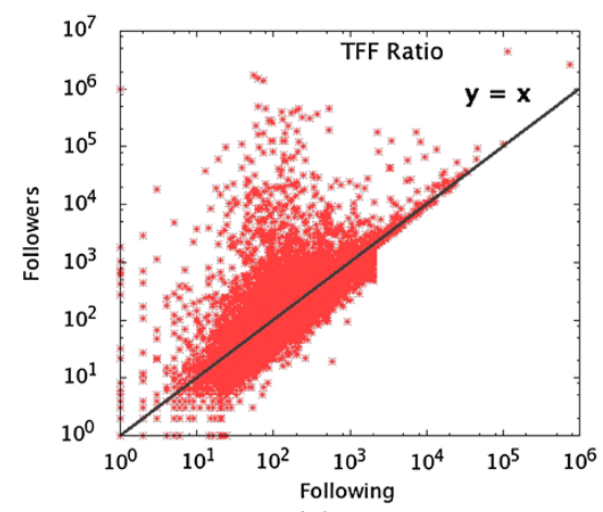

(b)

Fig. 2 (a) CCDF of followers and following, and (b) TFF

\subsection{Dataset characteristics}

We have built two collections, for the experiments. The first one, regards soda brands, contains 8,063 tweets, posted between August 2009 and September 2009, by 6,885 Brazilian users. The second one regards home appliance brands, has 2,354 tweets, posted between July and August 2010, by 1,671 users. All tweets are in Brazilian Portuguese. Next, we present some statistics for the dataset and why we believe they indicate that the method is generalizable.

\subsubsection{Generalization}

It is worth noticing that these topic-specific datasets have similar characteristics to previously analyzed samples of the Twitter network that are not restricted to a topic [18, 22, 23]. Such fact is shown in Fig. 2, with plots for the soda dataset. We analyzed the distribution of following and followers in a complementary cumulative distribution function (CCDF). In statistics and probability theory, CCDF describes the probability of a given value $a$ for taking a value above a particular level [19]. That is, $\bar{F}(x)=P(X>x)$. The $y$-axis of Fig. 2(a) represents the CCDF probability. The square points represent "following" while circles represent "followers" for the soda dataset. This distribution, specially the region beyond $x=10^{4}$, has a similar behavior to the one reported
Table 2 Tweets and users per sentiment

\begin{tabular}{llllll}
\hline & & + & 0 & - & Total \\
\hline \multirow{2}{*}{ soda } & Tweets & 3,083 & 4,156 & 824 & 8,063 \\
& Users & 2,770 & 3,401 & 714 & 6,885 \\
\multirow{3}{*}{ appliance } & Tweets & 1,489 & 580 & 285 & 2,354 \\
& Users & 1,198 & 360 & 149 & 1,707 \\
\hline
\end{tabular}

by Kwak et al. in [23]. This "stair-like behavior" shows that there's is a lack of users that follow and are followed by more than $10^{4}$ profiles. The similarities between the subjectrestricted dataset and the other generic samples of Twitter show that there are correspondent types of user in both contexts, which represent important indications that our method can be expanded to a wider context.

Also, Fig. 2(b) shows the follower/following ratio distribution among the users. It is possible to identify each type of user, according to the aforementioned Twitter FollowerFollowee Ratio on this plot: high ratio users ( $\uparrow$ followers, $\downarrow$ following) appear in the region above the diagonal; users with ratio approximately 1 (followers $\simeq$ followees) are around the $y=x$ line; and users whose ratio approaches zero ( $\downarrow$ followers, $\uparrow$ followees) are located below the diagonal. By comparing this TFF plot with previous work, such as [22], there are fewer representatives of the last group. Since their tweets are usually classified as noise (they may contain the keywords but often have unrelated advertising associated) and the set of users is built from the posted tweets, their representation in this dataset is smaller than usual. In order to be an influential user, the person must be an author: she must tweet.

The same analysis was conducted with the appliance dataset. The characteristics are similar; however, it presents sparser data and, for the following-follower plot there are more users around the line $y=x$. This occurs due to the particularities of the dataset: the subject is certainly less popular than the one in soda's dataset and most of the users are regular customers using Twitter as customer care platform.

\subsubsection{Other statistics}

According to the methodology for sentiment analysis (described in Sect. 3.1), each tweet of both datasets was manually classified as positive, negative, neutral or noise (if the tweet does not correspond to the respective topic) by a marketing and communication team of specialists. The specialists responsible for the tweet's classification are native speakers of Brazilian Portuguese (the dataset language). Table 2 presents the number of tweets and users for the datasets along with the respective sentiment classification. The soda dataset has a majority of neutral tweets, whereas the appliance one has a majority of positive. Soda brands are more 
Table 3 Statistics for $G_{i}$ and $G_{c}$ for both datasets

\begin{tabular}{llcl}
\hline & Nodes & $\operatorname{Arcs}$ in $G_{i}$ & $\operatorname{Arcs}$ in $G_{c}$ \\
\hline soda & 6885 & 797 & 8473 \\
appliance & 1707 & 1009 & 6103 \\
\hline
\end{tabular}
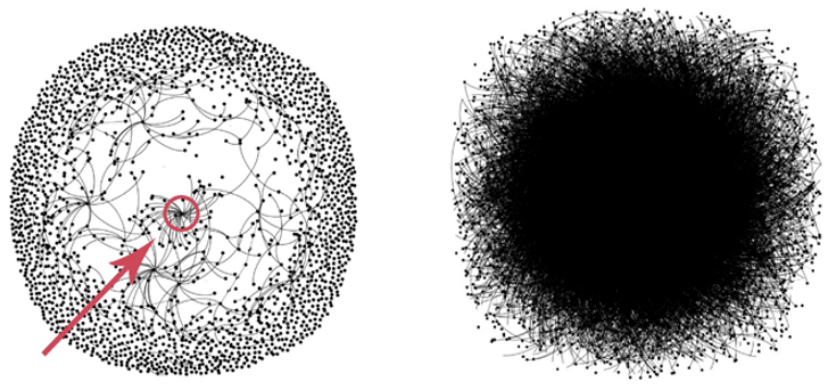

Fig. 3 Graphic representation of $G_{i}$ and $G_{c}$ for a soda dataset. The marked node in $G_{i}$ is a teen celebrity whose comment generated a large number of replies, as represented by the edges pointing to the node

present in people's routine than appliance brands. That is, soda brands may be cited in tweets that do not specifically talk about soda. This does not happen so frequently with appliance brands and, for that reason, tweets tend to be more polarized.

Table 3 compares the number of vertices and arcs of both graphs $G_{i}$ and $G_{c}$ built based on soda and appliance dataset and Fig. 3 displays a visual representation of both graphs for soda dataset. As shown in [18] (and visible in Fig. 3), the graph of interaction is considerably more sparse than the connection graph for both datasets. Accordingly, the number of arcs in $G_{c}$ is much larger than in $G_{i}$ in the two cases. ${ }^{10}$

\subsubsection{Influential users: ground truth}

Finally, for testing SaID, the marketing and communication specialists team created a list of influential users for the datasets. The procedure was analogous to the one for sentiment classification: at least two analysts classified each user as influent or not, and a supervisor checked the results, handling the disagreements. The claimed intuition was that users whose content was widespread, whose tweets were engaged toward a point of view and whose importance among the topic was relevant, were influential. They analyzed information about the tweets (RTs, replies) and the user (who she is, what types of tweet she usually writes, what the repercussion of her tweets was and so on). It is important to remark

\footnotetext{
${ }^{10}$ There may be connections that are not represented in $G_{c}$, due to changes in the user profile. Users may change their usernames or protect their accounts during the experiments, making it unavailable to collect their data. We expect these changes to be not significative, though.
}

that the same team analyzed both tweet sentiment and user influence.

For the soda dataset, they found 17 influential users: 10 evangelists and 7 detractors. Meanwhile, for the appliance dataset, they found 39 influential users: 23 evangelists and 16 detractors. No limit was imposed to the analysts in terms of maximum number of influential users per data set. Although the quantity of users found influent seems small, the team is used to this type of analysis and usually provides such service commercially.

\subsection{Experiments}

This section discusses the experiments aiming to validate and evaluate SaID. The experiments are divided into three main parts. First, we perform a detailed comparative analysis using paired observations of two branches of the method: one using the Interaction Graph and the other one using the Connection Graph. Second, we analyze the impact of each perspective (network, polarity and quality) on influential users' detection. Finally, we discuss the overall results for both evangelists and detractors.

\subsubsection{Experiment setup}

In order to evaluate our method, we employ ranking performance measures [2], assuming the specialists' influential lists as ground truth. The measures precision and recall were adjusted to the context of detecting influential users, as shown in (5) and (6), in which $n_{r}, n_{\text {ir }}$ and $n_{\text {it }}$ are: the number of users in the method's ranked list, the number of influential users in the method's ranked list and the total number of influential users in the dataset.

precision $=\frac{n_{\mathrm{ir}}}{n_{r}}$,

recall $=\frac{n_{\mathrm{ir}}}{n_{\mathrm{it}}}$.

Based on these two measures, we calculate the F-score, $\mathcal{F}_{\beta}$, of each rank as defined by (7). This measure can be interpreted as a weighted average of precision and recall.

$\mathcal{F}_{\beta}=\left(1+\beta^{2}\right) \times \frac{\text { precision } \times \text { recall }}{\left(\beta^{2} \times \text { precision }\right)+\text { recall }}$.

SaID was designed to assist social analysts on the monitoring task by providing a list of TOP- $x$ evangelists and detractors. As a manner of measuring its quality according to the ranked list size available, we evaluate our results using what we call [measure] @ $x$, meaning the measure (precision, recall or $\mathcal{F}_{\beta}$ ) value at a user ranked list of size $x$. The earliest (the shortest ranked list size) the method reaches the measures' maximum value, the higher is its performance. Therefore, our goal is to optimize each [measure] @ $x$ curve, 
considering $10 \leq x \leq 150$. We evaluate this, by calculating the area below the curve, for which we use the notation $a([$ measure $] @ x)$.

As claimed by the specialists, the number of influential users in a dataset is usually small when compared to the total of users. Due to this fact, although high precision is desired, it is far more valuable to evaluate whether the method is able to find all the influential users or not. For that matter, we focus on maximizing recall @ $x$. Also, we employ $\beta=2$ in our $\mathcal{F}_{\beta}$ evaluations $\left(\mathcal{F}_{2}\right.$ weights recall higher than precision).

Finally, two baselines were implemented for evaluating SaID. We call them naive models, due to their characteristics, defined as follows:

- Polarity Random Baseline, PRB, in which two random lists of users are generated: one for positive users and one for negative users.

- Polarity Ordered Baseline, $P O B$, in which two ranked lists of users are generated: one for positive users and one for negative users. The both lists ordered by the number of tweets posted by the user.

The measures recall @ $x$ and $\mathcal{F}_{2} @ x$ presented for the random model $(P R B)$ were calculated as the mean of $n$ samples, where for each dataset $n=\max \left(n_{x}^{i}\right), 0 \leq x \leq 150$ and $i=\{e, d\}$ (evangelists and detractors). The sample size $n_{x}^{i}$ was determined as the smallest sample size that provides an accuracy of $\pm 20 \%$, with a confidence level of $80 \%$, for the metric, at configurations $x$ and $i$, as described in [19]. We used 100 samples to estimate each $n_{x}^{i}$.

For recall @ $x$, we found $n=6000$ for both datasets and for $\mathcal{F}_{2} @ x, n=2800$ for the appliance dataset and $n=1000$ for the soda one. The high number of repetitions needed is a consequence of the small number of influential users. For example, considering the soda dataset, one influential accounts for $5.88 \%$ of the influential users set (1/17), leading to a high standard deviation, and consequently to a large number of samples needed for the given confidence and error.

\subsubsection{Interaction $\times$ Connection Graph}

For comparing the approaches, two types of influential users ranked list were generated for each dataset: one using the Interaction Graph $\left(G_{i}\right)$ and the other using the Connection Graph $\left(G_{c}\right)$ as source for the topology features calculation. As for the parameters $\alpha, \beta$ and $\gamma$, we used the combination that produced a rank with the best curve for recall @ $x$. A linearly independent set of $\alpha, \beta$ and $\gamma$ varying from 1 to 10 was tested. ${ }^{11}$

\footnotetext{
${ }^{11} \mathrm{~A}$ discussion about the parameters optimization and the impact of each perspective in the result will be held in Sect. 4.2.3.
}

Table $4 \mathcal{F}_{2}$ values for the ranked lists. The arrows indicate the higher (best) $(\boldsymbol{\Lambda})$ and lower (worst) $(\boldsymbol{\nabla})$ values. The circle $(\bullet)$ indicates equal or approximated values. The parameters $\alpha, \beta$ and $\gamma$ used in this experiment were: $(1,2,3)$ for soda connection, $(1,9,3)$ for soda interaction, $(1,9,1)$ for appliance connection and $(1,9,1)$ for appliance interaction

\begin{tabular}{|c|c|c|c|c|c|}
\hline & & $\mathcal{F}_{2}^{e}$ & $\mathcal{F}_{2}^{d}$ & $a\left(\mathcal{F}_{2}^{e}\right)$ & $a\left(\mathcal{F}_{2}^{d}\right)$ \\
\hline \multirow[t]{4}{*}{ soda } & $G_{i}$ & $1.00 \Delta$ & $0.05 \boldsymbol{\Lambda}$ & $1051.00 \boldsymbol{\Delta}$ & $95.00 \Delta$ \\
\hline & $G_{c}$ & $0.05 \mathrm{\nabla}$ & $0.04 \mathbf{\nabla}$ & $84.00 \mathrm{~V}$ & $85.00 \mathrm{~V}$ \\
\hline & POB & $0.03 \boldsymbol{\nabla}$ & $0.05 \bullet$ & $58.00 \boldsymbol{\nabla}$ & $31.00 \mathrm{~V}$ \\
\hline & PRB & $0.01 \nabla$ & $0.04 \bullet$ & $14.76 \mathbf{\nabla}$ & $43.65 \mathrm{~V}$ \\
\hline \multirow[t]{4}{*}{ appliance } & $G_{i}$ & $0.52 \boldsymbol{\Delta}$ & $0.11 \bullet$ & 510.43 ॥ & $169.00 \mathrm{\nabla}$ \\
\hline & $G_{c}$ & $0.07 \boldsymbol{\nabla}$ & $0.11 \bullet$ & $136.00 \nabla$ & $173.00 \Delta$ \\
\hline & POB & $0.07 \bullet$ & $0.11 \bullet$ & $117.00 \nabla$ & $73.00 \mathbf{\nabla}$ \\
\hline & PRB & $0.06 \nabla$ & $0.10 \nabla$ & $59.21 \boldsymbol{\nabla}$ & $71.32 \boldsymbol{\nabla}$ \\
\hline
\end{tabular}

Table 4 shows the $\mathcal{F}_{2}^{\{e, d\}}$ values for the generated ranked lists (evangelists and detractors for each graph used). The absolute values are calculated at ranked lists of size $x=150$. The area values $a\left(\mathcal{F}_{2}^{\{e, d\}}\right)$ are calculated for $10 \leq x \leq 150$. For the soda dataset, all the values for Interaction Graph are higher than the ones for Connection Graph. The values for the naive models were lower than both graph approaches, except for $\mathcal{F}_{2}^{d}$, whose values were the same. For the appliance dataset, the difference between the interaction and connection approaches is more subtle. The interaction one is better for two cases, equal to the connection in one and worse in one. This difference will be further explored in the next experiment. The naive models performance for the appliance dataset was worse than SaID, as happened for the soda dataset.

Next, we provide a deeper comparison of the ranked lists generated using the Connection and Interaction Graph approaches. For this analysis, we employ a common procedure called comparison of alternatives using paired observations [19]. This procedure compares two or more systems in order to find the best among them. The observations are called paired when, for two systems $A$ and $B$, in the $n$ experiments conducted, there is a one-to-one correspondence between the $i$ th test in system $A$ and the $i$ th test in system $B$. The two samples, generated by the experiments on $A$ and $B$, are treated as one sample of $n$ pairs. The difference of performance is computed for each pair and a confidence interval is defined. The interval is used as means of checking if the difference measured is significantly different from zero, at a desired level of confidence. If it is, the systems are significantly different. The sign indicates which one has a better performance.

We apply this procedure for comparing both approaches in the two datasets. We conducted 15 evaluations (recall @ $x$, $10 \leq x \leq 150$ ) consisting of paired observations of the experiments. The goal is to compare how many evangelists and detractors were retrieved using each approach, while 


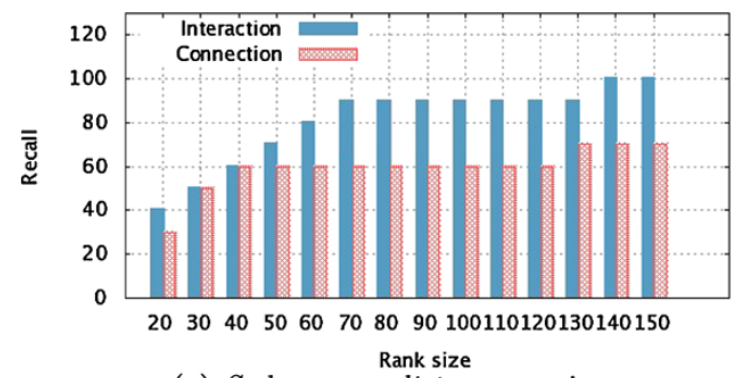

(a) Soda: evangelists comparison.

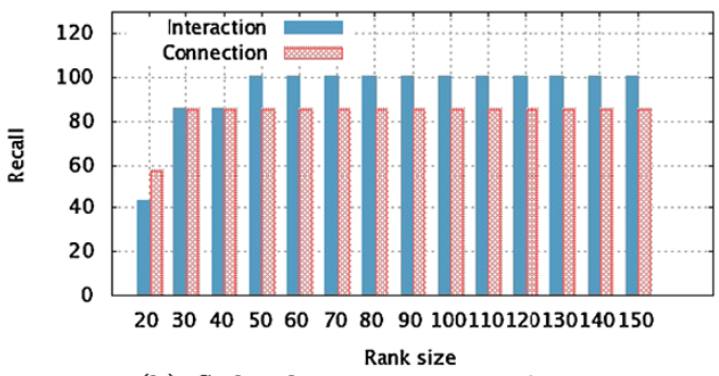

(b) Soda: detractors comparison.

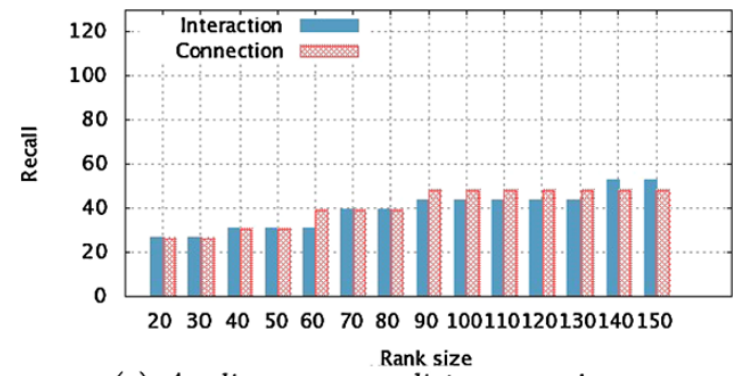

(c) Appliance: evangelists comparison.

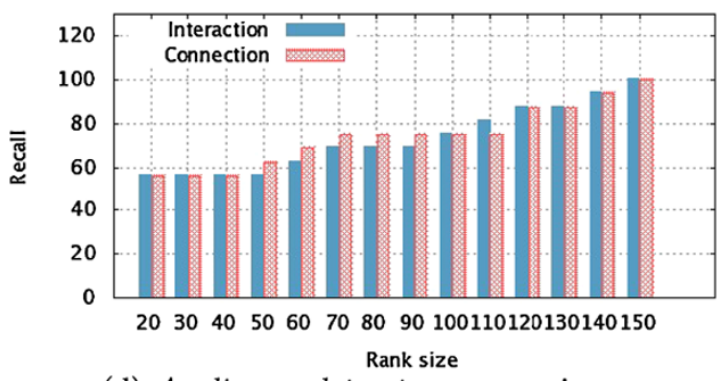

(d) Appliance: detractors comparison.

Fig. 4 Paired observations for Interaction and Connection Graph approaches for evangelists and detractors' recall @ $x$ in both datasets. The parameters $(\alpha, \beta, \gamma)$ of (4) are optimized for each scenario: soda + interaction: $(1,9,3)$; soda + connection: $(1,2,3)$; appliance + interaction: $(1,9,1)$; appliance + connection: $(1,9,1)$

the size of the ranked lists grows. We treat the samples of Interaction and Connection Graph as one single sample with 15 pairs and compute the difference for each one of them.

Figure 4 presents the values of evangelist's and detractor's recall @ $x$ for each approach and dataset. Table 5 presents the confidence interval of the recall difference for each option. The intervals were calculated with $95 \%$ of confidence. The Interaction Graph leads to better results in the
Table 5 Confidence intervals of recall difference (interactionconnection), with $90 \%$ of confidence

\begin{tabular}{lll}
\hline & evangelists & detractors \\
\hline soda & $(5.5147,13.5329)$ & $(14.3002,25.6998)$ \\
appliance & $(-3.0634,0.1648)$ & $(-3.3536,0.02028)$
\end{tabular}

Table 6 Computing time comparison, in seconds, of betweenness and eigenvector centrality in $G_{i}$ and $G_{c}$. The arrows indicate the higher (worst) ( $\mathbf{\Lambda}$ ) and lower (best) ( $\mathbf{\nabla})$ values

\begin{tabular}{llrl}
\hline & \multicolumn{1}{c}{$b c$} & \multicolumn{1}{l}{$e c$} \\
\hline soda & $G_{i}$ & $0.00(0.00) \boldsymbol{\nabla}$ & $1.96(0.21) \boldsymbol{\nabla}$ \\
& $G_{c}$ & $123.17(5.34) \boldsymbol{\Delta}$ & $8.84(0.40) \boldsymbol{\Delta}$ \\
appliance & $G_{i}$ & $0.00(0.00) \boldsymbol{\nabla}$ & $2.04(0.18) \boldsymbol{\nabla}$ \\
& $G_{c}$ & $96.32(3.13) \boldsymbol{\Delta}$ & $5.63(0.50) \boldsymbol{\Delta}$ \\
\hline
\end{tabular}

majority of scenarios. In the cases in which the Interaction approach is not better, the difference between the two approaches is not statistically significant (the interval includes 0 ), which means that they lead to approximately the same result. We believe that both graph-based approaches have similar results in the appliance dataset due to its smaller size. Since there are less users involved in the discussions about the brand, the chance of an interaction happen between two users that are connected is higher. As seen in Table 3, the number of arcs in $G_{i}$ and $G_{c}$ are similar to the ones for the soda dataset.

We also analyze the computational complexity of the extraction of betweenness $(b c)$ and eigenvector centrality $(e c)$ for $G_{i}$ and $G_{c}$, in each dataset. Each metric was calculated 10 times for each network and Table 6 exhibits the average mean cost and the standard deviation obtained (both in seconds). As expected, given the number of vertices and arcs shown in Table 3, the cost to compute features in $G_{i}$ is lower for both datasets. $G_{i}$ expresses only the real content-based connections between users reducing the problem complexity.

Based on these results, we conclude that the interaction based approach is better than the connection based one. For the soda dataset, $G_{i}$ produced better results with less computational cost. For the appliance dataset, even though the results were similar for both approaches, the interaction one is still cheaper. It is important to remind the reader that another additional cost of $G_{c}$ approach is to collect all the follower and following relations for the users in the dataset. Twitter API has limits of access, turning the pre-processing part slow and expensive.

\subsubsection{Parameters analysis}

The second part of the experiments aims to discuss the issues related to the parameters used in (4), $\alpha, \beta$ and $\gamma$. Also, 


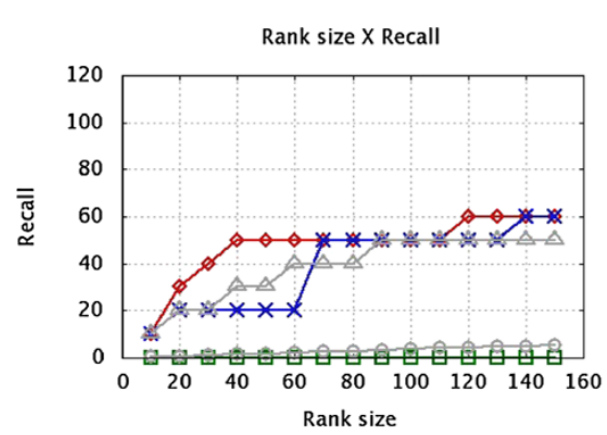

(a) Soda - evangelists

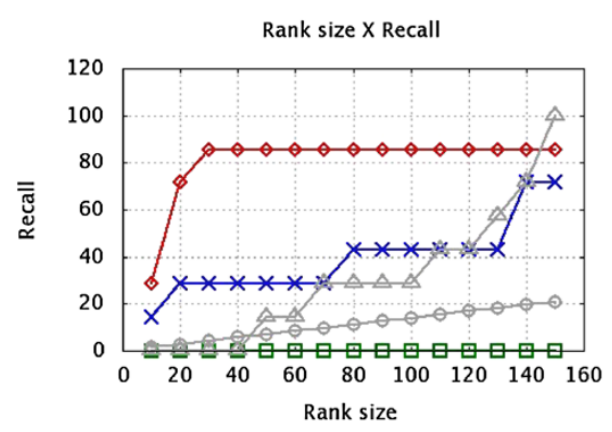

(b) Soda - detractors

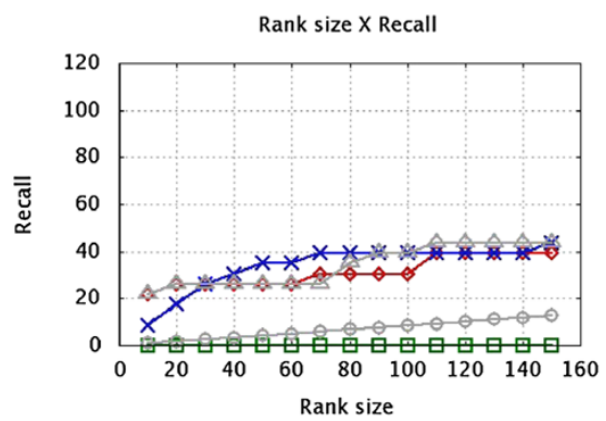

(c) Appliance - evangelists

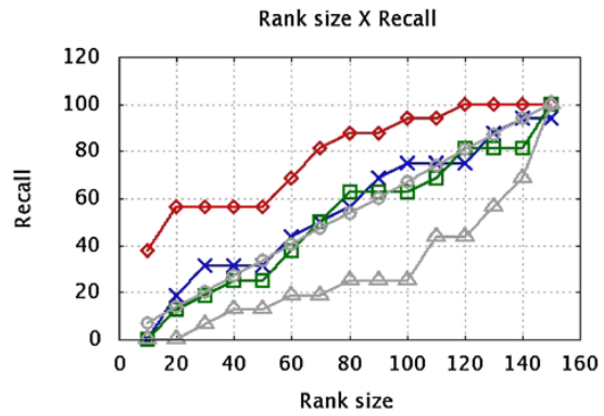

(d) Appliance - detractors

Fig. 5 Plot of recall @ $x$, using $G_{i}$, considering only polarity, network and quality in both datasets. For polarity the parameters of (4) are be $\alpha=1, \beta=\gamma=0$, for network, $\beta=1, \alpha=\gamma=0$ and for polarity, $\gamma=1, \alpha=\gamma=0$. naive model curves are also displayed for each case, for comparison we analyze the impact of each perspective in the method's result.

As stated before, a single view (polarity, network or quality) may not be good enough to classify users as influential or not. In order to test this hypothesis, different rankings were generated using only one component of (4) at a time. Figure 5 presents recall @ $x$ results for each isolated component using both datasets. We also present values for the two aforementioned naive models.

As can be seen, polarity by itself gives better results than the other perspectives on detractors detection for both datasets. This happens mainly due to the smaller quantity of negative tweets (and users) and the facility with which negative tweets are identifiable. Our polarity factor also outperforms both naive models presented. For similar reasons, the network perspective works better for evangelists: besides the larger volume of positive tweets, analysts claim that the difference between neutral and positive tweets is quite subtle (which can lead to errors if one looks only at the polarity). Comparing the network factor the naive models the ordered method POB has a similar performance to the network factor alone most of the time. The network factor does not take into account the positive or negative bias of the user, which is very important for the polarized detection, and is partially covered by POB. Finally, the quality perspective, alone, does not help on detecting neither the evangelists nor the detractors on soda dataset. This happens also for detractors detection for the appliance dataset. We believe that the low performance of the quality perspective is probably due to the informal and noisy vocabulary used by Twitter users. On the other hand, for detractors identification in the appliance dataset, quality by itself is practically as good as the network perspective. As already mentioned, in the appliance dataset most of the negative tweets are from users who explore Twitter as customer care platform, reporting problems and dissatisfactions directly to the official brand profile. For such reason, We believe that the negative tweets are significantly well-written.

In order to perform a deeper analysis of the impact that each perspective has on the final method results, we employ a $2^{k}$ experimental (or factorial) design [19].

In a experimental design, the outcome of an experiment is called the response variable and is the manner of measuring the system performance. Each variable that affects the response variable and has different alternatives is called a factor or predictor and its alternatives (the values it can assume) are called levels. A full factorial design investigates every possible combination at all levels of all factors, determining the effect of $k$ different factors (and inter-factor interactions) on the response variable. The number of factors and their levels can be very large and, consequently, the full factorial design may be expensive. Thus, there is a very popular design, called $2^{k}$ design, in which each of the 
Table 7 Factorial design results for both evangelist (E) and detractors (D) for both datasets

\begin{tabular}{|c|c|c|c|c|c|c|c|c|}
\hline \multicolumn{9}{|c|}{ Factorial design results } \\
\hline Soda & Factors & $A$ & $B$ & $C$ & $A B$ & $A C$ & $B C$ & $A C B$ \\
\hline $\mathrm{D}$ & $\%$ variation & $87.20 \% \boldsymbol{\Delta}$ & $5.60 \% \bullet$ & $2.17 \% \nabla$ & $2.21 \%$ & $0.33 \%$ & $2.14 \%$ & $0.34 \%$ \\
\hline $\mathrm{E}$ & $\%$ variation & $41.26 \% \Delta$ & $22.55 \% \bullet$ & $7.02 \% \nabla$ & $9.53 \%$ & $9.86 \%$ & $6.91 \%$ & $2.87 \%$ \\
\hline Appliance & Factors & $A$ & $B$ & $C$ & $A B$ & $A C$ & $B C$ & $A B C$ \\
\hline $\mathrm{D}$ & $\%$ variation & $60.41 \% \boldsymbol{\Delta}$ & $9.05 \% \boldsymbol{\nabla}$ & $23.21 \% \bullet$ & $0.04 \%$ & $1.01 \%$ & $6.29 \%$ & $0.00 \%$ \\
\hline E & $\%$ variation & $49.91 \%$ & $13.94 \%$ & $13.28 \% \nabla$ & $5.50 \%$ & $8.83 \%$ & $5.93 \%$ & $2.62 \%$ \\
\hline
\end{tabular}

$k$ factors is evaluated at two levels. This design acts as a preliminary investigation of which factors are relevant for a deeper investigation. The importance of a factor is measured by the proportion that it explains of the total variation of the response and, in particular, the factors which explain a high percentage of variation are considered the most relevant for further investigation. The steps of an illustrative factorial design with two factors $A$ and $B$ can be summarized as follows.

\section{$2^{k}$ Factorial design steps}

1. Each of their $k$ factors is associated to variables $x_{A}$ and $x_{B}$, which stand for the lower and higher levels, as follows:

$$
x_{k}= \begin{cases}-1 & \text { if factor } k \text { assumes its lower level, } \\ +1 & \text { if factor } k \text { assumes its higher level. }\end{cases}
$$

2. The performance (response variable) $y$ of systems $A$ and $B$ are regressed on $x_{A}$ and $x_{B}$ using a nonlinear regression model of the form: $y=q_{0}+q_{A} x_{A}+q_{B} x_{B}+$ $q_{A B} x_{A} x_{B}$.

3. The effects $q_{0}, q_{A}, q_{B}$ and $q_{A B}$ are determined by expressions called contrasts, which are linear combinations of the responses $y_{i}$ calculated based on observations of each possible combinations of the variables. If $x_{A i}$ and $x_{B} i$ are the levels of $x A$ and $x B$, respectively, the observation would be modeled as $y_{i}=q_{0}+q_{A} x_{A i}+q_{B} x_{B i}+$ $q_{A B} x_{A i} x_{B i}$.

4. The importance of a factor is measured by the proportion of the total variation in the response that is explained by the factor. In order to calculate this proportion, it is first, necessary to calculate the total variation of $y$, or the sum of squares of total, given by $S S T=\sum_{i=1}^{2^{k}}\left(y_{i}-\bar{y}\right)^{2}$.

5. Also, $S S T$ can be expressed as $S S T=2^{k} q_{A}^{2}+2^{k} q_{B}^{2}+$ $2^{k} q_{A B}^{2}$. The three parts on the right-hand side represent the portion of the total variation explained by the effect of $A, B$, and interaction $A B$, such as $S S A=2^{k} q_{A}^{2}, S S B=$ $2^{k} q_{B}^{2}$ and so on. Thus, the fraction of variation explained by a factor $k$ is given by $k=\frac{S S k}{S S T}$. Finally, this fraction provides means to gauge the importance of the factor.
For our experiment, we define the variables $x_{A}$, for polarity, $x_{B}$, for network, and $x_{C}$, for quality and the response variable is $a$ (recall @ $x$ ) for, $10 \leq x \leq 150$. The combination of factors was the following:

$x_{A}= \begin{cases}-1 & \text { if } \alpha=\frac{1}{\left|u_{\text {polarity }}\right|}, \\ +1 & \text { if } \alpha=1,\end{cases}$

$x_{B}=\left\{\begin{array}{ll}-1 & \text { if } \beta=0, \\ +1 & \text { if } \beta=1,\end{array} \quad x_{C}= \begin{cases}-1 & \text { if } \gamma=0, \\ +1 & \text { if } \gamma=1 .\end{cases}\right.$

For polarity, in the lowest level, only the signal of user's polarity is considered, while for the highest, the intensity is also taken into account. For example, considering a user with polarity perspective $u_{\text {polarity }}=-12$, in the lowest level (replacing $\alpha$ in the influence score formula, (4), the polarity part would be

$$
\begin{aligned}
\alpha \times u_{\text {polarity }} & =\frac{1}{\left|u_{\text {polarity }}\right|} \times u_{\text {polarity }} \\
& =\frac{1}{12} \times(-12) \\
& =-1 .
\end{aligned}
$$

Meanwhile, in the highest level, the polarity part would be $\alpha \times u_{\text {polarity }}=1 \cdot u_{\text {polarity }}=-12$. For the network and quality perspectives, the levels were defined as the presence or absence of the component in the influence score formula ( $\beta=\{0,1\}$ and $\gamma=\{0,1\})$. The intuition of employing this design is to analyze what is the effect on the results when a perspective can be left out.

In total, four scenarios were studied for each dataset, applying the described experimental design. In the first two (D1 and D2), we considered the retrieval of detractors, the next two (E1 and E2), the retrieval of evangelists. Table 7 shows the results for each of the six designs by means of the fraction of variation for each factor for the datasets. The perspective that turns out to be the most responsible for the variation either worsens or improves the results with much more intensity than the others. 
Fig. 6 Ternary plot of $\alpha, \beta$ and $\gamma$ values for the Interaction Graph method. Each combination of parameters is a circle. The color (from a grayscale palette) represents the value for the area below the recall@x curve: $a($ recall $)$

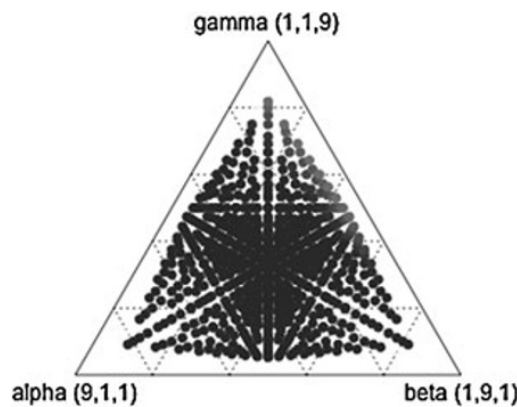

(a) Soda dataset

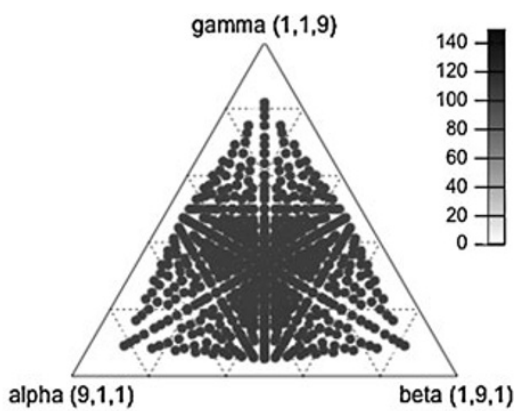

(b) Appliance dataset
By observing the results, we can conclude that the responsible for the greatest fraction of the variation of results in both datasets is the polarity factor. The use of the polarity signal, instead of its intensity, worsens the result largely. Also, as observed in Fig. 5, the polarity is one of the most important perspectives in the method. The other two perspectives behave differently for the different datasets. For the soda dataset, quality is the minor responsible for the variation for both evangelists and detractors. This means that the presence or absence of the metric does not impact the method much, that is, its contribution for influence detection is small. Meanwhile, for the appliance dataset, quality was responsible for a fraction of variation similar (evangelists) or greater (detractors) than the network perspective. Looking at both datasets, the network factor stays between the other two perspectives, except for detractors detection for the appliance dataset. Observing the corresponding plot in Fig. 5, it is possible to conclude that this happens due to the good results using any of the three perspectives alone (including the quality one): once all the perspectives play a important role in the detection, the fraction of variation is distributed more fairly.

Finally, determining the best combination of $\alpha, \beta$, and $\gamma$ is an issue. For the reported experiments, we have optimized the parameters by searching linearly all the combinations from 1 to 10 . Due to the small number of influential users in each dataset and the impossibility to employ methods such as leave one out [1] (we want to evaluate the rank, not each user), we optimized the parameters using the whole data, in order to estimate the potential of the method. Although limitations are expected from this methodology of optimization, Fig. 6 shows that the result does not change much for different values of $\alpha, \beta$ and $\gamma$. Specifically, in the ternary plots, each edge corresponds to a parameter and its values increase vertically according to its opposite base. Each point is a combination of the three of parameters. The color of each point indicates the area below the recall @ $x$ curve $a$ (recall) for the combination of parameters that it represents. The scale, from 0 , white, to 150 , black is also shown.
By analyzing the plots, one can see that the values of recall@ $x$ are only slightly affected by the change of parameter combination for both datasets. Moreover, the result range for both datasets is similar: around $a($ recall @ $x) \sim$ 100. Therefore, when dealing with a new dataset, a choice of parameters that is similar to the ones presented in this work is expected to produce good results as well.

\subsubsection{Evangelists vs. detractors}

Finally, in this Section, we aim to discuss the final results for evangelists and detractors using the Interaction-based approach. Figure 7 shows recall @ $x$ for evangelists and detractors. We also display the naive models for comparison purposes.

In both datasets, the result is better for detractors than for evangelists. This difference is mainly because it is easier for an analyst to classify a detractor; it is usually difficult to differ between positive and neutral tweets, which may lead to more errors on finding the evangelists. Furthermore, comparing the results presented in Fig. 5 (using only one perspective at a time) and Fig. 7 (using the combination of the perspectives), one can see that the latter usually produces better ranked lists than the former for both datasets. In the appliance dataset, for example, although the polarity curve for detractors is very similar to the one produced by combining the perspectives, it does not produce good results for evangelists, when compared to the combination of the perspectives. An ideal curve is one that detects the highest number of influential users as quick as possible, and, for that matter, the combined curve is the best choice.

As to the naive model curves, all of them are outperformed by SaID. It is interesting to note that the random plots (PRB) produce straight lines: influential users or not influential ones are added progressively to the rank. Also, for the appliance dataset, the random model for detractors (PRB-d) is better than the ordered one (POB-d). This indicates that the number of tweets per user is not really related to its classification as detractor. Other factors have to be considered, for example, network and quality as in our method. 


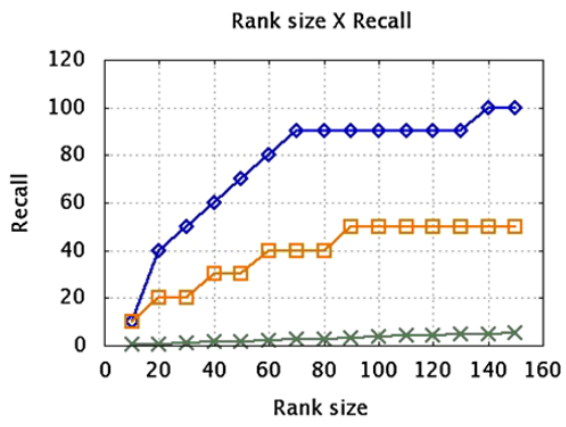

(a) Soda dataset - evangelists

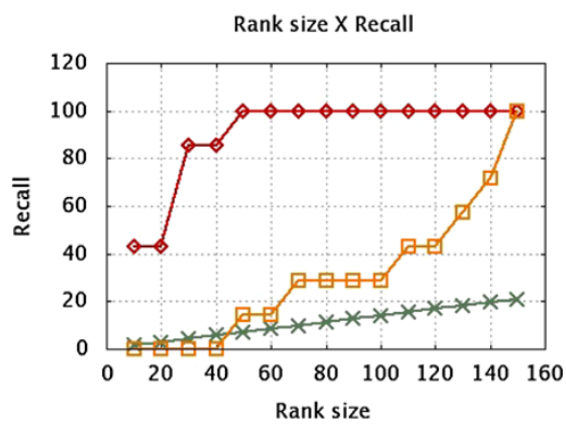

(b) Soda dataset - detractors

Rank size X Recall

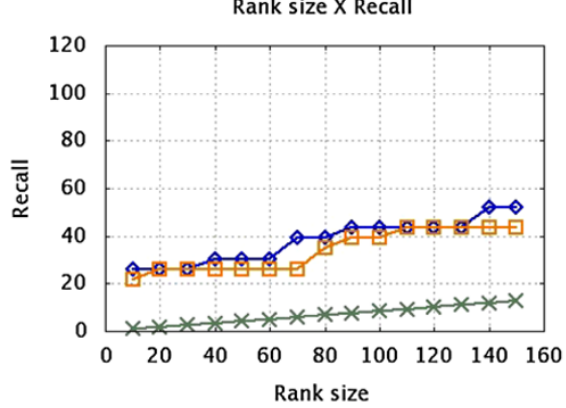

(c) Appliance - evangelists

Rank size X Recall

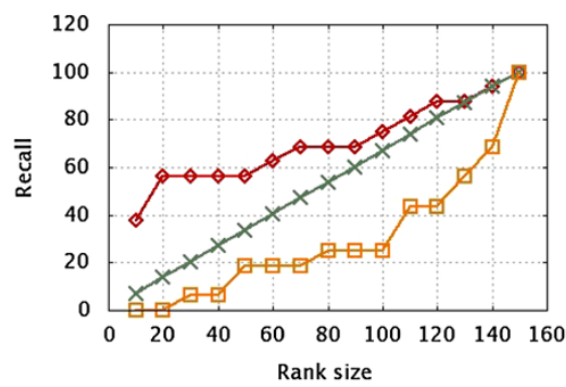

(d) Appliance - detractors

Fig. 7 recall @ $x$ for evangelists and detractors. The parameters $\alpha$, $\beta$ and $\gamma$ of (4) are $(1,9,3)$ for soda dataset and $(1,9,1)$ for appliance dataset. $P O B_{e}, P O B_{d}, P R B_{e}$ and $P R B_{d}$ are, respectively, Polarity Ordered Baseline for evangelists and detractors and Polarity Random Baseline for evangelists and detractors

\section{Conclusion}

In this article, we addressed the problem of identifying biased influential users on a topic in Twitter. Motivated by the dynamics of this environment, in which users share opinions, experiences and suggestions about diverse subjects, and by the huge volume of content generated daily, we aim to assist businesses (or anyone interested in product/service feedback) on finding the key users that lead the conversations and actions for a given subject.

This work has analyzed user behavior, interaction and connections in order to determine their influence on Twitter. Specifically, for each user, her tweets' readability and polarity are extracted, and her position in two different networks (Interaction and Connection Network) of people that talk about the same topic are analyzed. Moreover, since there is no benchmark for influential users detection (a default dataset with tweets and users previously classified), one significant effort of this work was to build such a test collection. This is not a trivial task due to the difficulty to classify the tweet's sentiment and the user's level of influence (both subjective problems by nature).

We have validated our method using specialists' ground truth for two product datasets, studied the impact of each perspective on influential identification, and compared the results using Interaction and Connection Networks. We have found that the detractor's result is visibly more accurate than the evangelist's. This happens due to the occasional difficulty for distinguishing between a neutral and a positivebiased tweet during the manual classification. For the negative tweets, this boundary is usually clearer. The experimental results also demonstrated that the interactions (mentions, replies, re-tweets, attributions) of an user with others is a better representation of her influence than her connections (follower, following). The recall values for the generated ranks, using the interactions, were always better. Another substantial remark is that the Interaction Network is more sparse than the Connection one. This means more accurate results with cheaper computational cost.

As future work, we plan to implement and test a full automatic approach of SaID, as well as improve the parametrization of polarity, network and quality factors. To include temporal aspects in influential detection is also planned. Finally, we aim to expand our experiments in more datasets, featuring different characteristics.

Acknowledgements This work is partially supported by the projects INCT-Web (MCT/CNPq grant 57.3871/2008-6) and by the authors' individual grants and scholarships from CNPq, CAPES and FAPEMIG.

\section{References}

1. Alpaydin E (2004) Introduction to machine learning (adaptive computation and machine learning). MIT Press, Cambridge

2. Baeza-Yates RA, Ribeiro-Neto B (1999) Modern information retrieval. Addison-Wesley, Reading

3. Bakshy E, Hofman JM, Mason W, Watts DJ (2011) Everyone's an influencer: quantifying influence on twitter. In: International conference on web search and data mining (WSDM), Hong Kong, China 
4. Barabási A-L (2002) Linked: the new science of networks, 1st edn. Basic Books, New York

5. Berry J, Keller E (2003) The influentials: one American in ten tells the other nine how to vote, where to eat, and what to buy. Free Press, New York

6. Bonacich P (2007) Some unique properties of eigenvector centrality. Soc Netw 29(4):555-564

7. Brandes U (2008) On variants of shortest-path betweenness centrality and their generic computation. Soc Netw 30(2):136-145

8. Brin S, Page L (1998) The anatomy of a large-scale hypertextual web search engine. Comput Netw ISDN Syst 30:107-117

9. Cha M, Haddadi H, Benevenuto F, Gummadi KP (2010) Measuring user influence in Twitter: the million follower fallacy. In: Conference on weblogs and social media, Washington, District of Columbia, USA

10. Chan KK, Misra S (1990) Characteristics of the opinion leader: a new dimension. J Advert 19:53-60

11. Chen P, Xie H, Maslov S, Redner S (2007) Finding scientific gems with Google's PageRank algorithm. J Informetr 1(1):8-15

12. Costa LF et al (2007) Characterization of complex networks: a survey of measurements. Adv Phys 56:167

13. Dalip DH et al (2009) Automatic quality assessment of content created collaboratively by web communities: a case study of Wikipedia. In: Joint conference on digital libraries (JCDL), Austin, Texas, USA, pp 295-304

14. Golbeck J, Hansen D (2011) Computing political preference among Twitter followers. In: Proceedings of the 2011 annual conference on human factors in computing systems, CHI '11, Vancouver, British Columbia, Canada. ACM, New York, pp 1105-1108

15. Goyal A, Bonchi F, Lakshmanan LV (2010) Learning influence probabilities in social networks. In: International conference on web search and data mining (WSDM), New York, New York, USA, pp 241-250

16. Hagberg A, Schult D, Swart P Networkx. High productivity software for complex networks. https://networkx.lanl.gov/

17. Huang J, Thornton KM, Efthimiadis EN (2010) Conversational tagging in Twitter. In: Conference on hypertext and hypermedia, Toronto, Ontario, Canada, pp 173-178

18. Huberman BA, Romero DM, Wu F (2008) Social networks that matter: Twitter under the microscope. Social science research network working paper series
19. Jain RK (1991) The art of computer systems performance analysis: techniques for experimental design, measurement, simulation, and modeling. Wiley/Interscience, New York

20. Jansen BJ et al (2009) Twitter power: tweets as electronic word of mouth. J Am Soc Inf Sci Technol 60(11):2169-2188

21. Katz E, Lazarsfeld P, CUB of Applied Social Research (1955) Personal influence: the part played by people in the flow of mass communications. Foundations of communications research. Free Press, New York

22. Krishnamurthy B, Gill P, Arlitt M (2008) A few chirps about Twitter. In: Workshop on online social networks (WOSP), Seattle, Washington, USA, pp 19-24

23. Kwak H, Lee C, Park, H, and Moon S (2010) What is Twitter, a social network or a news media. In: International conference on World Wide Web (WWW), Raleigh, North Carolina, USA.

24. Leavitt A, Burchard E, Fisher D, Gilbert S (2009) The influentials: new approaches for analyzing influence on Twitter

25. Lee C, Kwak H, Park H, Moon S (2010) Finding influentials based on the temporal order of information adoption in Twitter. In: International conference on World Wide Web (WWW), Raleigh, North Carolina, USA, pp 1137-1138

26. O'Connor B, Balasubramanyan R, Routledge BR, Smith NA (2010) From tweets to polls: linking text sentiment to public opinion time series. In: International AAAI conference on weblogs and social media (ICWSM), Washington, District of Columbia, USA

27. Ressler S (1993) Perspectives on electronic publishing: standards, solutions, and more

28. Ruhnau B (2000) Eigenvector-centrality—a node-centrality? Soc Netw 22(4):357-365

29. Savage N (2011) Twitter as medium and message. Commun ACM $54: 18-20$

30. Van den Bulte C, Joshi YV (2007) New product diffusion with influentials and imitators. Mark Sci 26(3):400-421

31. Watts DJ, Dodds PS (2007) Influentials, networks, and public opinion formation. J Consum Res 34(4):441-458

32. Weng J, Lim EP, Jiang J, He Q (2010) Twitterrank: finding topicsensitive influential twitterers. In: International conference on web search and data mining (WSDM), New York, New York, USA, pp 261-270 